\title{
Decoupling effects in the running of the Cosmological Constant
}

\section{Oleg Antipin}

Rudjer Bošković Institute, Division of Theoretical Physics, Bijenička 54, HR-10000 Zagreb, Croatia

E-mail: oantipinairb.hr

\section{Blaženka Melić *}

Rudjer Bošković Institute, Division of Theoretical Physics, Bijenička 54, HR-10000 Zagreb,

\section{Croatia}

E-mail: melicairb.hr

\begin{abstract}
We study the decoupling effects associated with heavy particles in the renormalization group running of the vacuum energy in a mass-dependent renormalization scheme. We find the running of the vacuum energy stemming from the Higgs condensate in the entire energy range and show that it behaves as expected from the simple dimensional arguments meaning that it exhibits the quadratic sensitivity to the mass of the heavy particles in the infrared regime. The consequence of such a running to the fine-tuning problem with the measured value of the Cosmological Constant is analyzed and the constraint on the mass spectrum of a given model is derived. This finetuning constraint is not satisfied in the Standard Model (SM) while in the massless theories it formally coincides with the well known Veltman condition. We also provide a remarkably simple extension of the SM where saturation of this constraint enables us to predict the radiative Higgs mass correctly. Generalization to constant curvature spaces is also given.
\end{abstract}

EPS-HEP 2017, European Physical Society conference on High Energy Physics 5-12 July 2017

Venice, Italy

\footnotetext{
* Speaker.
} 


\section{Introduction}

We start by briefly reviewing the Cosmological Constant (CC) problem and for this purpose it is sufficient to work in the low energy domain of the gravitational physics so that we start with the bare Hilbert-Einstein action with coupling constants $G_{v a c}, \Lambda_{v a c}$ and $\xi$ :

$$
S_{H E}=\int d^{4} x \sqrt{-g}\left\{\mathscr{L}_{S M}+\xi^{0} \varphi^{\dagger} \varphi R-\frac{1}{16 \pi G_{v a c}^{0}}\left(R+2 \Lambda_{\text {vac }}^{0}\right)\right\} .
$$

The bare quantities are defined with the superscript " 0 ". Let us focus on the various contributions to the vacuum energy density defining

$$
\left(\rho_{\Lambda}^{0}\right)^{v a c}=\frac{\Lambda_{v a c}^{0}}{8 \pi G_{v a c}^{0}} \quad \rho_{\text {ind }}^{0}=V\left(\varphi_{v a c}\right)=-\frac{m^{4}}{2 \lambda}
$$

where the "induced" contribution $\rho_{\text {ind }}^{0}$ to the vacuum energy density is arising from the vacuum condensates i.e. for $\varphi_{v a c}$ being the value of the Higgs field $\varphi(x)$ which minimizes the Higgs potential $V(\varphi)=-m^{2} \varphi^{\dagger} \varphi+\frac{\lambda}{2}\left(\varphi^{\dagger} \varphi\right)^{2}, \rho_{\text {ind }}^{0}$ is the Higgs condensate contribution (at the classical level) to the induced vacuum energy. Both contributions to the vacuum energy must be renormalized so that $\rho_{\Lambda}^{\mathrm{vac}}(\mu)$ and $\rho_{\text {ind }}(\mu)$ and the connection with experimentally measured value $\rho_{\text {phys }}$ at $\mu_{c}=\mathscr{O}\left(10^{-3}\right) \mathrm{eV}$ has to be achieved. Besides the vacuum and induced terms we may have additional effects from the higher derivative gravitional terms as well as corrections from quantum gravity so that the physical value $\rho_{\text {phys }}$ measured at the cosmological RG scale $\mu_{c}$ finally reads

$$
\rho_{\text {phys }}=\rho_{\Lambda}^{\mathrm{vac}}\left(\mu_{c}\right)+\rho_{\text {ind }}\left(\mu_{c}\right)+\ldots=10^{-47} \mathrm{GeV}^{4} .
$$

The problem is that if we use the experimental Higgs mass $m_{H}=125 \mathrm{GeV}$, then the corresponding value $\left|\rho_{\text {ind }}\left(\mu \sim v_{e w} \approx 246 \mathrm{GeV}\right)\right| \sim v_{e w}^{4} \simeq 10^{8} \mathrm{GeV}^{4}$. Therefore, neglecting all the ... terms in (1.3), the $\rho_{\Lambda}^{\mathrm{vac}}$ and $\rho_{\text {ind }}$ should cancel with the precision of 55 decimal orders. This is the CC fine-tuning problem [2].

To deeper understand this tuning, one has to also take into account the decoupling effects of massive particles due to the RG running from the high energies to the present one, $\mu_{c}=\mathscr{O}\left(10^{-3}\right)$ $\mathrm{eV}$. Clearly, we expect that contribution to the RG running from the particle of mass $m$ should change dramatically as we go from $\mu \gg m$ to $\mu \ll m$ regime. This leads to additional contribution to $\rho_{\text {ind }}(\mu)$ with the leading term scaling as $\mu^{2} m^{2}$. We will show that requiring the absence (or, at least, reduction) of this additional tuning due to $\mu_{c}^{2} m^{2}$ term may provide a constraint on the spectrum of the particle physics models [1].

\section{RG running of the Cosmological Constant in the Standard Model}

\subsection{Mass-independent $(\overline{M S})$ scheme}

The renormalized effective potential $V$ of the SM can be written in the 't Hooft-Landau gauge and the $\overline{M S}$ scheme as [3]

$$
\begin{aligned}
V\left(\rho_{\Lambda}^{\mathrm{vac}}, \phi, m^{2}, \lambda_{i}, \mu\right) & \equiv V_{0}+V_{1}+\cdots, \\
V_{0}=-\frac{1}{2} m^{2} \phi^{2}+\frac{1}{8} \lambda \phi^{4}, \quad V_{1} & =\sum_{i=1}^{5} \frac{n_{i}}{64 \pi^{2}} M_{i}^{4}(\phi)\left[\log \frac{M_{i}^{2}(\phi)}{\mu^{2}}-c_{i}\right]+\rho_{\Lambda}^{\mathrm{vac}}
\end{aligned}
$$


Table 1: Contributions to the effective potential (2.1) from the SM particles $W^{ \pm}, Z^{0}$, top quark t, Higgs $\phi$ and the Goldstone bosons $\chi_{1,2,3}$.

\begin{tabular}{|c||ccccc|}
\hline$\Phi$ & $i$ & $n_{i}$ & $\kappa_{i}$ & $\kappa_{i}^{m}$ & $c_{i}$ \\
\hline$W^{ \pm}$ & 1 & 6 & $g^{2} / 4$ & 0 & $5 / 6$ \\
$Z^{0}$ & 2 & 3 & $\left(g^{2}+g^{2}\right) / 4$ & 0 & $5 / 6$ \\
\hline $\mathrm{t}$ & 3 & -12 & $y_{\mathrm{t}}^{2} / 2$ & 0 & $3 / 2$ \\
\hline$\phi$ & 4 & 1 & $3 \lambda / 2$ & 1 & $3 / 2$ \\
\hline$\chi_{i}$ & 5 & 3 & $\lambda / 2$ & 1 & $3 / 2$ \\
\hline
\end{tabular}

where $\lambda_{i} \equiv\left(g, g^{\prime}, \lambda, h_{t}\right)$ runs over all dimensionless couplings and $V_{0}, V_{1}$ are the tree level potential and the one-loop correction respectively. The tree-level background masses $M_{i}^{2}(\phi)=\kappa_{i} \phi^{2}-\kappa_{i}^{m} m^{2}$ of the particles with coefficients $n_{i}, \kappa_{i}, \kappa_{i}^{m}$, and $c_{i}$ defined in Table. 1 enter in the one-loop radiative corrections and $M_{1} \equiv m_{W}, M_{2} \equiv m_{Z}, M_{3} \equiv m_{t}, M_{4} \equiv m_{\text {Higgs }}, M_{5} \equiv m_{\text {Goldstone }}$.

We split the effective potential into two pieces: the $\phi$-independent (vacuum) term and the $\phi$-dependent "scalar" term

$$
V\left(\rho_{\Lambda}^{\mathrm{vac}}, \phi, m^{2}, \lambda_{i}, \mu\right)=V_{\text {vac }}\left(\rho_{\Lambda}^{\mathrm{vac}}, m^{2}, \lambda_{i}, \mu\right)+V_{\text {scal }}\left(\phi, m^{2}, \lambda_{i}, \mu\right) .
$$

The RG-invariance of the full renormalized effective potential reads (with $\gamma_{m} m^{2}=\beta_{m^{2}}$ ):

$$
\left(\mu \frac{\partial}{\partial \mu}+\beta_{\lambda_{i}} \frac{\partial}{\partial \lambda_{i}}+\gamma_{m} m^{2} \frac{\partial}{\partial m^{2}}-\gamma_{\phi} \phi \frac{\partial}{\partial \phi}+\beta_{\rho_{\Lambda}^{\mathrm{vac}}} \frac{\partial}{\partial \rho_{\Lambda}^{\mathrm{vac}}}\right) V\left(\rho_{\Lambda}^{\mathrm{vac}}, \phi, m^{2}, \lambda_{i}, \mu\right)=0,
$$

and it is possible to show that Eq. (2.4) is, in fact, a sum of two independent RG equations,

$$
\begin{aligned}
& \left(\mu \frac{\partial}{\partial \mu}+\beta_{\lambda_{i}} \frac{\partial}{\partial \lambda_{i}}+\gamma_{m} m^{2} \frac{\partial}{\partial m^{2}}+\beta_{\rho_{\Lambda}^{\text {vac }}} \frac{\partial}{\partial \rho_{\Lambda}^{\text {vac }}}\right) V_{\text {vac }}\left(\rho_{\Lambda}^{\text {vac }}, m^{2}, \lambda_{i}, \mu\right)=0, \\
& \left(\mu \frac{\partial}{\partial \mu}+\beta_{\lambda_{i}} \frac{\partial}{\partial \lambda_{i}}+\gamma_{m} m^{2} \frac{\partial}{\partial m^{2}}-\gamma_{\phi} \phi \frac{\partial}{\partial \phi}\right) V_{\text {scal }}\left(\phi, m^{2}, \lambda_{i}, \mu\right)=0 .
\end{aligned}
$$

It is straightforward to check that the requirement (2.4) applied to the full one-loop effective potential (2.1) leads to the conditions

$$
\frac{1}{8} \beta_{\lambda}-\frac{1}{2} \gamma_{\phi} \lambda=\sum_{i} \frac{n_{i} \kappa_{i}^{2}}{32 \pi^{2}}, \quad \frac{1}{2} \gamma_{m}-\gamma_{\phi}=\sum_{i} \frac{n_{i} \kappa_{i} \kappa_{i}^{m}}{16 \pi^{2}}, \quad \mu \frac{\partial \rho_{\Lambda}^{\mathrm{vac}}}{\partial \mu}=m^{4} \sum_{i} \frac{n_{i}\left(\kappa_{i}^{m}\right)^{2}}{32 \pi^{2}}
$$

up to two-loop corrections. The first two equations come from (2.6) and the last condition satisfies (2.5). These equations show explicitly that the vacuum $V_{v a c}$ and scalar $V_{\text {scal }}$ parts of the full effective potential satisfy independent RG equations. Combining (2.7) appropriately we finally obtain

$$
\mu \frac{\partial\left(\rho_{\Lambda}^{\mathrm{vac}}+\rho_{\text {ind }}\right)}{\partial \mu}=m^{4}\left(\sum_{i} \frac{n_{i} \kappa_{i}^{2}}{8 \pi^{2} \lambda^{2}}-\sum_{i} \frac{n_{i} \kappa_{i} \kappa_{i}^{m}}{8 \pi^{2} \lambda}+\sum_{i} \frac{n_{i}\left(\kappa_{i}^{m}\right)^{2}}{32 \pi^{2}}\right)=\sum_{i} \frac{n_{i}}{32 \pi^{2}} M_{i}^{4}(\langle\phi\rangle),
$$

where we used

$$
\rho_{\text {ind }}(\mu) \equiv V_{0}(\langle\phi\rangle)=-\frac{m^{4}(\mu)}{2 \lambda(\mu)} \quad M_{i}^{2}(\langle\phi\rangle)=\kappa_{i}\langle\phi\rangle^{2}-\kappa_{i}^{m} m^{2}=m^{2}\left(\kappa_{i} \frac{2}{\lambda}-\kappa_{i}^{m}\right)
$$

and $\langle\phi\rangle^{2}=2 m^{2} / \lambda$. Eq.(2.8) is the central equation valid in the $U V$ regime of massless and massive theories, theories with the spontaneous symmetry breaking (SSB) and without. 


\subsection{Decoupling effects of heavy particles}

By definition, the RG equation (2.8) holds in the region $\mu \gg m$ and to go to the opposite regime $\mu \ll m$ would require to take into account: 1$)$ the contribution of heavy particles at the energies near their mass, 2) the residual effects from the heavy particles at energies well below their mass.

It is well-known that the decoupling of heavy particles does not hold in a mass-independent scheme like the $\overline{M S}$, and for this reason they must be decoupled by hand using the sharp cut-off procedure or some of the mass-dependent schemes. The quantum effects of the massive particles are, in principle, suppressed at low energies by virtue of the Appelquist-Carazzone theorem, so that in the region below the mass of the particle its quantum effects become smaller. On purely dimensional grounds, in the regime $\mu \ll m$ one expects the corrections to the CC of the type $\mu^{2} m^{2}$. These corrections can be seen from the fact that in a mass-dependent subtraction scheme a heavy mass $m$ enters the $\beta$-functions through the dimensionless combination $\mu / m$, so that the $\mathrm{CC}$, being a dimension- 4 quantity, is expected to have the $\beta$-function corrected as follows:

$$
\beta\left(m_{\text {light }}, \frac{\mu}{m}\right)=a m_{\text {light }}^{4}+b\left(\frac{\mu}{m}\right)^{2} m^{4}+c\left(\frac{\mu}{m}\right)^{4} m^{4}+\ldots
$$

where $a, b$ and $c$ are some coefficients, $m_{\text {light }}$ is some light mass $m_{\text {light }} \ll \mu$, and the dots stand for terms suppressed by higher order powers of $\mu / m \ll 1$. We will now show how one can deal with the decoupling effect in the SM and how to calculate explicitly the coefficients $a, b, c$ for any model.

\subsection{Mass-dependent scheme}

In the $\overline{M S}$ renormalization scheme, one chooses counterterms (c.t.) in such a way as to remove the divergent $\sim 1 / \varepsilon$ pole and scale independent number $-\gamma_{E}+\log (4 \pi)$ in the scattering amplitudes and therefore, by construction, the counterterms are mass-independent. In a mass-dependent renormalization scheme, the counterterms are mass-dependent and can be chosen, for example, to subtract, in addition to the divergent pole and scale-independent numbers above, also the value of the amplitude evaluated at the external momentum $p^{2}=-\mu^{2}$. After this additional finite subtraction, (2.8) will be replaced by the corresponding expression in the momentum subtraction scheme (MOM) which reads [1]

$$
\mu \frac{\partial\left(\rho_{\Lambda}^{\mathrm{vac}}+\rho_{\text {ind }}\right)}{\partial \mu}{ }_{\mid \mathrm{MOM}}=\sum_{i} \frac{n_{i}}{32 \pi^{2}} M_{i}^{4}(\langle\phi\rangle) \int_{0}^{1} \frac{x(1-x) \mu^{2} d x}{M_{i}^{2}(\langle\phi\rangle)+x(1-x) \mu^{2}} .
$$

This is the master equation describing the running of $\mathrm{CC}$ in any regime, non-decoupling and decoupling one, which is valid both in the $U V$ and the IR regime. Working in the region where $\left(m_{\text {light }}^{2}\right)_{j} \ll \mu^{2} \ll M_{i}^{2}(\langle\phi\rangle)$ where $M_{i}^{2}(\langle\phi\rangle)=\left(M_{t}^{2}, M_{W}^{2}, M_{Z}^{2}, M_{H}^{2}\right)$ are heavy SM particles, we obtain:

$$
\mu \frac{\partial\left(\rho_{\Lambda}^{\mathrm{vac}}+\rho_{\text {ind }}\right)}{\partial \mu}=\sum_{j} \frac{n_{j}\left(m_{\text {light }}^{4}\right)_{j}}{32 \pi^{2}}+\frac{\mu^{2}}{12(4 \pi)^{2}}\left[-12 M_{t}^{2}+6 M_{W}^{2}+3 M_{Z}^{2}+M_{H}^{2}\right]+\frac{\mu^{4}}{30(4 \pi)^{2}}+\ldots
$$

The above expression is exactly of the form of (2.10) and proves the expected decoupling behavior in the effective theories. The light masses $m_{\text {light }}$ may be, again, generated by the Higgs vev 
$m_{\text {light }}(\langle\phi\rangle)$ such as a mass for, say, charm quark, or may be a new mass parameters in the SM Lagrangian related, for example, to the neutrino masses. As the $\mu$-scale slides down the energy, more and more SM masses will migrate from the $m_{\text {light }}^{4}$-term to the inside of the brackets in the $\mu^{2}$-term. The $\mu^{2} M_{i}^{2}$ term in the running of $\rho_{\Lambda}^{\text {vac }}+\rho_{\text {ind }}$ provides the leading RG effect due to the heavy SM particles and we may demand it to vanish as to reduce the fine-tuning in the physical value of the $\mathrm{CC}$ at the $\mu_{c}=\mathscr{O}\left(10^{-3}\right) \mathrm{eV}$. This requirement, however, leads to the SM prediction $m_{H} \approx 550 \mathrm{GeV}$, inconsistent within the experimental value of $m_{H} \approx 125 \mathrm{GeV}$.

\section{RG running of the Cosmological Constant in the massless theories}

In the massless limit $m=0$, from (2.7) we have $\rho_{\Lambda}^{\text {vac }}=$ const and only $\rho_{\text {ind }}$ runs with $\mu$. Also, in these theories at the tree-level $\langle\phi\rangle=0$ which means that the tree-level mass of the Higgs is zero and the electroweak symmetry needs to be broken radiatively. For this to happen, we need to balance the tree-level potential against the 1-loop contribution, so that for consistent perturbative expansion we have to impose the value of the Higgs quartic couplings at the electroweak scale to be parametrically given as $\lambda \sim\left(g^{4}, g^{\prime^{4}}, y_{t}^{4}\right)$. This allows us to simplify (2.12) to obtain $(i=W, Z, t$ and neglecting the light masses $\left.m_{\text {light }}\right)$ :

$$
\mu \frac{\partial \rho_{\text {ind }}}{\partial \mu}=\frac{\langle\phi\rangle^{4}}{32 \pi^{2}} \sum_{i} n_{i} \kappa_{i}^{2} \int_{0}^{1} \frac{x(1-x) \mu^{2} d x}{\left(M_{\text {phys }}^{2}\right)_{i}+x(1-x) \mu^{2}}=\frac{\mu^{2}\langle\phi\rangle^{2}}{12(4 \pi)^{2}} \frac{\partial}{\partial \phi^{2}} \sum_{i} n_{i} M_{i}^{2}(\phi)+\frac{\mu^{4}}{20(4 \pi)^{2}}+\ldots
$$

where in the last line we used the fact that in the massless theory with only one background field $\phi$, any mass can be written as $M_{i}^{2}(\phi)=\kappa_{i} \phi^{2}$. Notice that the $\mu^{2}$-proportional term is nothing but the generalization of the well known Veltman condition i.e. the requirement of the absence of the quadratic divergence for the Higgs mass (cancellation of the prefactor of the $\phi^{2}$-term). This means that in the massless case the fine-tuning problem of the Higgs mass is linked to the fine-tuning problem of the Cosmological Constant value.

Let us consider the simplest extension of the SM by adding one extra massless real scalar $S$ :

$$
V_{0}=V_{0}^{S M}+\lambda_{H S} \Phi^{\dagger} \Phi S^{2}+\frac{\lambda_{S}}{4} S^{4}
$$

so that contribution from the Higgs background to the mass of the scalar $S$ is given by $M_{S}^{2}(\phi)=$ $\lambda_{H S} \phi^{2}$. In this model, the solution to the Veltman condition (3.1) reads

$$
12 M_{t}^{2}-6 M_{W}^{2}-3 M_{Z}^{2}-M_{S}^{2}=0 \Longrightarrow \lambda_{H S}(\mu)=6 y_{t}^{2}(\mu)-\frac{9}{4} g^{2}(\mu)-\frac{3}{4} g^{\prime 2}(\mu) \stackrel{\mu \approx v_{E W}}{\approx} 4.8 .
$$

Working in the parameter space of the model where $\langle S\rangle=0$, see [5] for details, leads to the scalar mass $M_{S}=\sqrt{\lambda_{H S}} v_{E W} \approx 550 \mathrm{GeV}$ which we already noticed above in the massive version of the SM where the role of the scalar $S$ was played by the Higgs.

$$
M_{H}^{2}=\frac{3}{8 \pi^{2}}\left[\frac{1}{16}\left(3 g^{4}+2 g^{2} g^{\prime 2}+g^{\prime 4}\right)-y_{t}^{4}+\frac{1}{3} \lambda_{H S}^{2}\right] v_{E W}^{2} \quad \Longrightarrow M_{H} \approx 125 \mathrm{GeV} .
$$

This provides an interesting example of how the demand for the absence of leading RG effects in the running of the $\rho_{\text {ind }}$ due to the heavy particles may provide the hints on the possible extensions of the SM. 


\section{Standard Model in the constant curvature space}

Generalizing the SM to the constant curvature space in the mass-dependent scheme we obtain [1]:

$$
\begin{aligned}
& \mu \frac{\partial\left(\rho_{\Lambda}^{\mathrm{vac}}+\rho_{\text {ind }}+\kappa R\right)}{\partial \mu}=\sum_{i} \frac{n_{i}}{32 \pi^{2}} \mathscr{M}_{i}^{4}(\langle\phi\rangle) \int_{0}^{1} \frac{x(1-x) \mu^{2} d x}{\mathscr{M}_{i}^{2}(\langle\phi\rangle)+x(1-x) \mu^{2}}= \\
& \sum_{j} \frac{n_{j}\left(\mathscr{M}_{\text {light }}^{4}\right)_{j}}{32 \pi^{2}}+\frac{\mu^{2}}{12(4 \pi)^{2}}\left[-12 \widetilde{m}_{t}^{2}+6 \widetilde{m}_{W}^{2}+3 \widetilde{m}_{Z}^{2}+\widetilde{m}_{H}^{2}+\frac{7}{3} R\right]+\frac{\mu^{4}}{30(4 \pi)^{2}}
\end{aligned}
$$

where masses $\widetilde{m}_{i}^{2}$ have corrections from the non-minimal Higgs coupling $\xi$

$$
\widetilde{m}_{i}^{2} \equiv M_{i}^{2}(\langle\phi\rangle)-2 \kappa_{i} \frac{\xi R}{\lambda}
$$

with $M_{i}^{2}(\langle\phi\rangle)$ defined in (2.9). The result (4.1) also generalizes effective theory expansion (2.10) to the constant curvature space

$$
\beta\left(m_{\text {light }}, \frac{\mu}{m}\right)=a_{1} m_{\text {light }}^{4}+b_{1} \mu^{2} m^{2}+c_{1} \mu^{4}+d_{1} \mu^{2} R+\ldots
$$

\section{Conclusions}

We revisited the decoupling effects associated with heavy particles in the RG running of the vacuum energy using the mass-dependent renormalization scheme. We derived the universal oneloop beta function of the vacuum energy $\rho_{\Lambda}^{\mathrm{vac}}+\rho_{\text {ind }}$, arising from the Higgs vacuum and the Cosmological Constant term in the entire energy range, valid in the UV and in the IR regime. We have shown that although $\rho_{\Lambda}^{\text {vac }}$ and $\rho_{\text {ind }}$ run separately, it is only the sum $\rho_{\Lambda}^{\text {vac }}+\rho_{\text {ind }}$ that exhibits behavior consistent with the decoupling theorem, in the SM as well as in massless theories with the simple extension and in the generalization at the constant curvature spaces.

\section{References}

[1] O. Antipin and B. Melic, "Revisiting the decoupling effects in the running of the Cosmological Constant," Eur. Phys. J. C 77 (2017) no.9, 583 [arXiv:1703.10967 [hep-th]].

[2] I. L. Shapiro and J. Sola, "On the possible running of the cosmological 'constant', "Phys. Lett. B 682 (2009) 105 [arXiv:0910.4925 [hep-th]].

[3] J. A. Casas, J. R. Espinosa and M. Quiros, "Improved Higgs mass stability bound in the standard model and implications for supersymmetry," Phys. Lett. B 342 (1995) 171 doi:10.1016/0370-2693(94)01404-Z [hep-ph/9409458].

[4] I. L. Shapiro and J. Sola, "Scaling behavior of the cosmological constant: Interface between quantum field theory and cosmology," JHEP 0202 (2002) 006 [hep-th/0012227].

[5] O. Antipin, M. Mojaza and F. Sannino, "Conformal Extensions of the Standard Model with Veltman Conditions," Phys. Rev. D 89 (2014) no.8, 085015 [arXiv:1310.0957 [hep-ph]]. 